\title{
Moving Mass Trim Control System Design ${ }^{1}$
}

\author{
Raymond H. Byrne \\ Sandia National Labs \\ MS 1125, PO Box 5800 \\ Albuquerque, NM 87185-1125 \\ rhbyrne@sandia.gov
}

\author{
Rush D. Robinett \\ Sandia National Labs \\ MS 00313, PO Box 5800 \\ Albuquerque, NM 87185-0310 \\ rdrobin@sandia.gov
}

\author{
Beverly Rainwater Sturgis \\ Sandia National Labs \\ MS 0303, PO Box 5800 \\ Albuquerque, NM 87185-0303 \\ brsturg@sandia.gov
}

\begin{abstract}
This paper describes the design of a moving mass trim control system for maneuvering axisymmetric reentry vehicles. The moving mass trim controller is composed of three equal masses that are independently positioned in order to deliver a desired center of mass position. For a slowly spinning reentry vehicle, the mass offset creates a trim angle-of-attack to generate modest flight path corrections. The control system must maintain the desired position of each mass in the face of large disturbances. A novel algorithm for determining the desired mass positions is developed in conjunction with a preliminary controller design. The controller design is based on classical frequency domain techniques where a bound on the disturbance magnitude is used to formulate the disturbance rejection problem. Simulation results for the controller are presented for a typical reentry vehicle.
\end{abstract}

\section{Introduction}

Over the years, techniques for controlling the flight characteristics of missiles and reentry vehicles (RV) have gravitated to systems that deliver relatively large amounts of control authority. For certain missions, such as an air-to-air missile or an RV designed to evade defenses, a large lateral acceleration capability is required. The technologies used to perform these missions range from actuated canards, elevons, and flaps to jet interaction, thrust vector control, and a variety of other techniques [1]. Because of inherent navigation inaccuracies, systems that provided modest amounts of control capabilities were of little or no value. However, with the maturity of the Global Positioning System (GPS), it is possible to mate simpler control techniques with GPS to increase the accuracy of existing systems. One such technology is a moving

\footnotetext{
${ }^{1}$ This work was supported by the United States Department of Energy under Contract DE-AC04-94AL8500
} 
mass controller. This technique has previously been evaluated in conjunction with other control methods such as the moving mass roll control of an aerodynamically asymmetric RV [2,3]. A more direct application of moving mass control technology is the moving mass trim controller (MMTC) [4]. The MMTC generates a trim angle-of-attack (AOA) on an axisymmetric, ballistic vehicle directly from the motion of the mass. For a slow spinning reentry vehicle, the MMTC generates a mass offset to create a trim AOA due to aerodynamic drag. This paper presents the design of a moving mass trim control system for reentry vehicle terminal guidance.

The guidance and control system for a reentry vehicle with a MMTC consists of several subsystems: the guidance scheme, the mass position algorithm, the moving masses, and the reentry vehicle dynamics. These are shown in Figure 1. The guidance scheme determines the corrective actions necessary to hit the target in the form of a commanded lateral acceleration or a commanded angle-of-attack. The moving mass controller determines the required mass positions from these commands and attempts to maintain these positions in the face of disturbances. Both the reentry vehicle and the moving masses are dynamic systems subject to disturbances and changes (uncertainty) over the course of the flight. This paper focuses on the design of the moving mass control system, which must determine the desired mass positions based on the commanded center of mass, and maintain these desired mass positions in the face of large disturbances. The mass position algorithm for placing three masses to obtain a desired center of mass is discussed in section

2. The expected disturbances are characterized in section 3. The controller design is presented in section 4. Simulation results for the moving mass control system are discussed in section 5 . Section 6 contains a summary and recommendations for future research.

\section{Mass Position Algorithm}

The mass position algorithm calculates the placement of the three masses to obtain a desired center of mass. Two masses may be used to generate any equivalent center of mass, but small movements in the desired center of mass can result in large mass movements. In a three mass system, small changes in the 


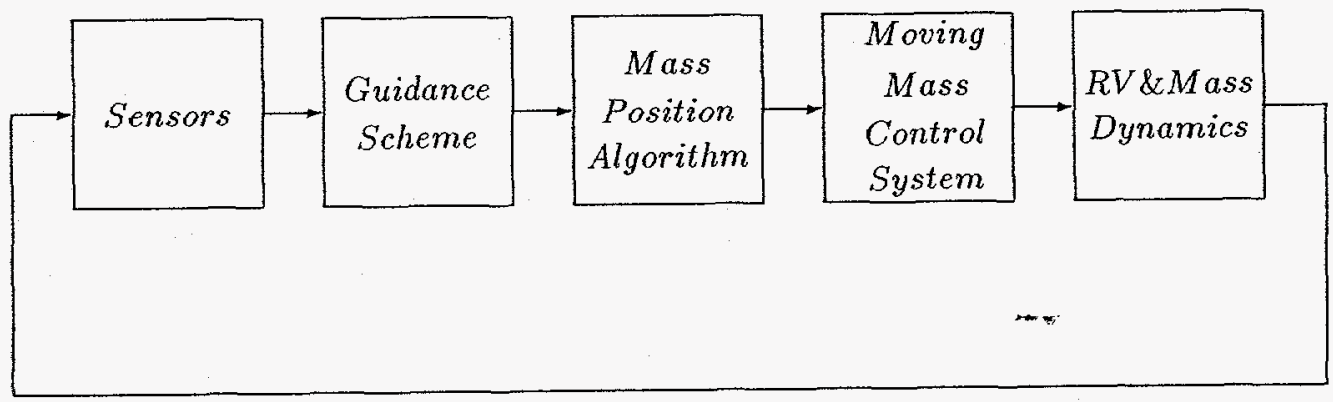

Figure 1: Simplified Diagram of Guidance and Control System

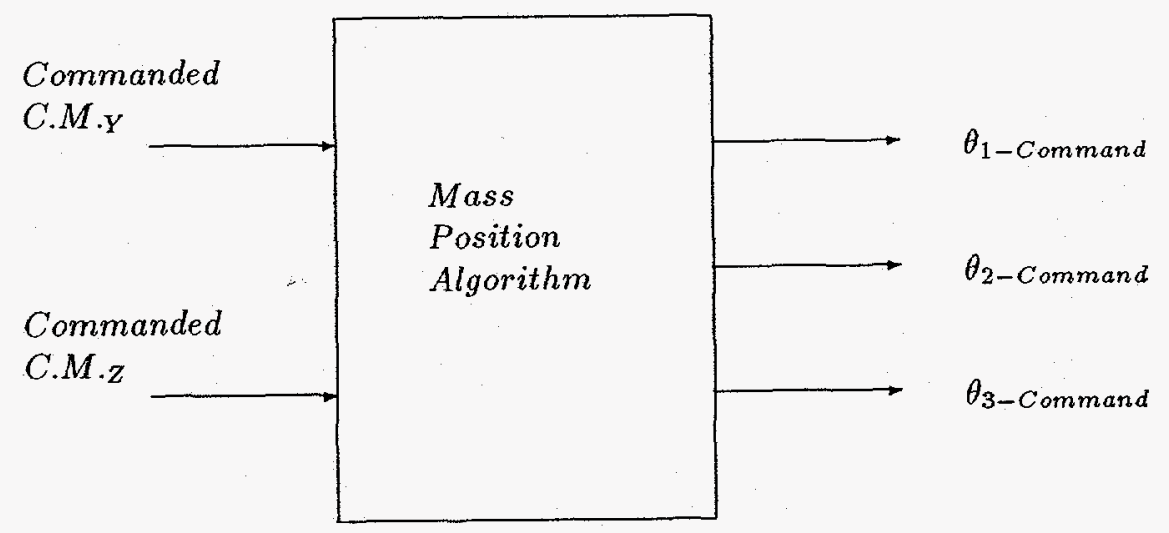

Figure 2: Mass Position Algorithm Block Diagram 


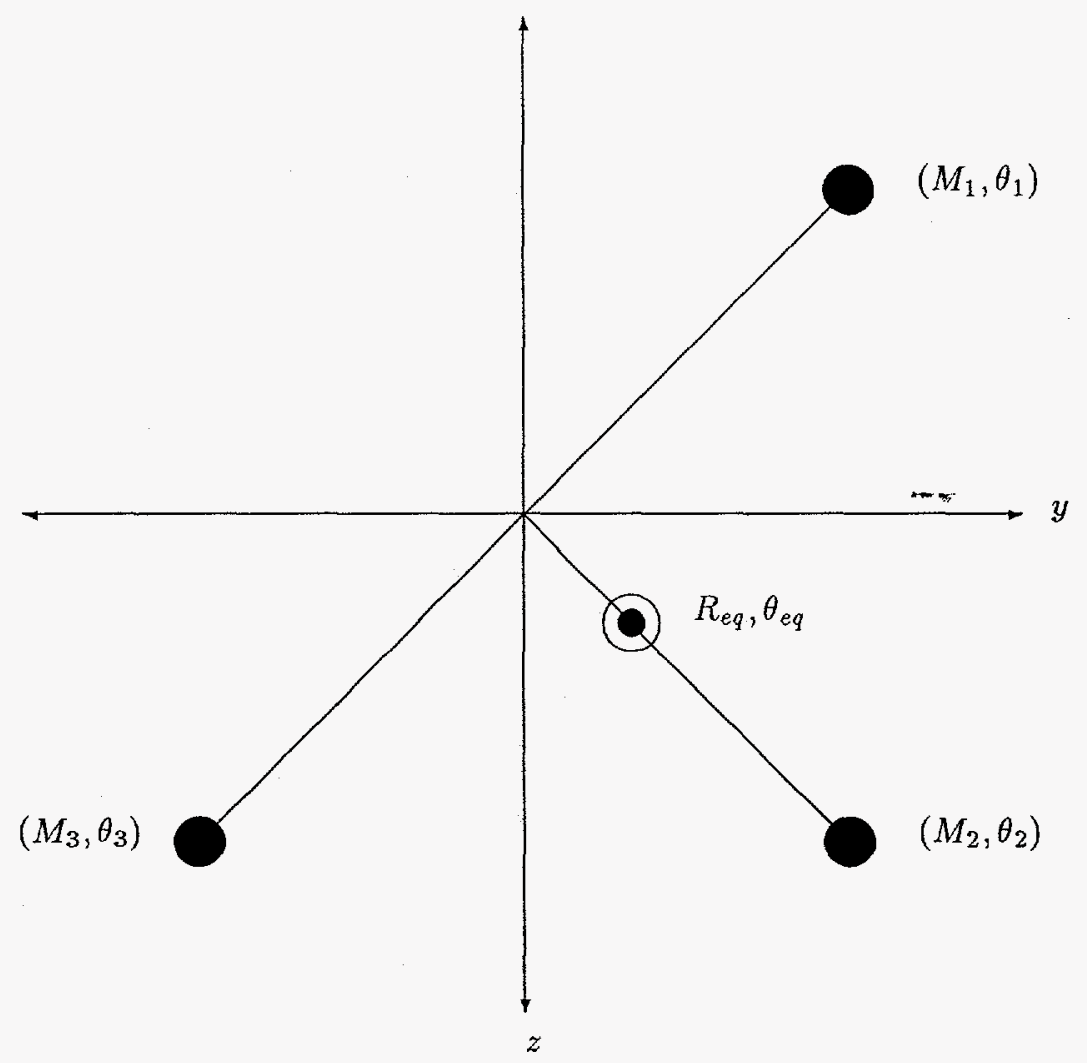

Figure 3: Three Mass System

commanded center of mass can be more easily handled with small changes in the position of each mass. This section describes an algorithm that determines the placement of the three masses to obtain a desired center of mass (see Figure 2).

The location of the center of mass, assuming three equal masses located at the polar coordinates $\left(R, \theta_{i}\right)$, is given by

$$
R_{e q} e^{j \theta_{e q}}=\frac{R}{3}\left(e^{j \theta_{1}}+e^{j \theta_{2}}+e^{j \theta_{3}}\right)
$$

where $R_{e q}$ and $e^{j \theta_{e q}}$ represent the polar coordinates of the resulting center of mass (c.m.). The three mass system is shown in Figure 3 .

A desirable performance objective is to minimize the movement of the masses required to obtain a new 
center of mass. Several ways to express this performance objective are listed below.

$$
\begin{gathered}
J=\left(\triangle \theta_{1}\right)^{2}+\left(\triangle \theta_{2}\right)^{2}+\left(\triangle \theta_{3}\right)^{2} \\
J=\sup \left|\triangle \theta_{i}\right|, \quad i=1,2,3
\end{gathered}
$$

Equation (2) represents a quadratic cost function, which minimizes the total movement, while equation (3) represents an $H^{\infty}$ type cost function which minimizes the maximum distance that any one mass will have to travel. Other possible performance objectives might include minimizing the net rotation of the masses to reduce the effects of the moving masses on the spin rate of the reentry vehicle. The problem of moving three masses to obtain a desired center of mass is an optimization problem with nonlinear constraints. This type of problem is very difficult to solve in a closed form. Therefore, one approach is to linearize the problem, and then solve for the optimal solution of the linearized equation.

Linearizing equation (1), one obtains the following equation for the movement of the center of mass.

$$
\left[\begin{array}{c}
\triangle y \\
\triangle z
\end{array}\right]=\frac{R}{3} H\left[\begin{array}{c}
\Delta \theta_{1} \\
\Delta \theta_{2} \\
\Delta \theta_{3}
\end{array}\right]
$$

where

$$
H=\left[\begin{array}{ccc}
-\sin \left(\theta_{1}\right) & -\sin \left(\theta_{2}\right) & -\sin \left(\theta_{3}\right) \\
\cos \left(\theta_{1}\right) & \cos \left(\theta_{2}\right) & \cos \left(\theta_{3}\right)
\end{array}\right]
$$

An "optimal" solution to equation (4), which minimizes the length of the $\Delta \theta$ vector, is shown in equation (6) $[5]$.

$$
\left[\begin{array}{c}
\triangle \theta_{1} \\
\triangle \theta_{2} \\
\triangle \theta_{3}
\end{array}\right]=\frac{3}{R} H^{T}\left(H H^{T}\right)^{-1}\left[\begin{array}{c}
\Delta y \\
\triangle z
\end{array}\right]
$$

Because equation (6) is a linearization, the incremental movements of the center of mass must be kept small. As a result, a large desired movement in the center of mass must be broken down into a trajectory of smaller movements. Simulation of the algorithm showed that it works well as long as the desired location 
of the center of mass is within a radius of $R / 3$, where $R$ is the radius of the three masses. Difficulties with the linearized algorithm arise when the commanded center of mass is outside the radius $R / 3$ or when the direction of movement of the desired center of mass is directly away from one of the masses. Also, certain orientations result in oscillations of the center of mass as well as the location of the three masses. Adding noise to the mass angles improves the case when moving the desired c.m. directly away from one of the masses, but the trajectory of the c.m. can still make large movements away from the desired trajectory.

- Several other optimal linearized solutions were also explored. All of the linearized optimal solutions suffered from the problems previously described. In order to overcome some of the limitations of the linearized algorithms, a hybrid algorithm was developed which uses the linearization for small radius commands and a heuristic algorithm for larger radius commands. The heuristic algorithm involves choosing the closest mass to the desired c.m. angle and moving that mass in line with the desired c.m. angle. By constraining the angle of one mass and spacing the other two masses evenly, the nonlinear equation (1) may be solved directly. Hysteresis is used to eliminate rapid algorithm switching. A state diagram of the mass position algorithm appears in Figure 4. Through simulations, suitable values of $R_{1}$ and $R_{2}$ were found to be $R_{1}=0.15 R$ and $R_{2}=0.2 R$.

\section{Disturbance Characterization}

Moving masses that generate a mass offset to create a trim AOA are subject to large disturbances. These disturbances are the result of accelerations in the plane of the masses. In addition, ablation effects and body roll-rate disturbances are also seen as torque disturbances in the plane of the masses. In order to quantify these disturbances, simulations which model them were run with the three masses held in one location. For the simulations, a $4.3 \mathrm{lbf}$ mass was used. The torques seen by each of the $4.3 \mathrm{lbf}$ masses is shown in Figure 5.

Looking at Figure 5, the maximum disturbance torque is approximately $3.5 f t-l b f$. An FFT of the disturbance torque was performed to determine the frequency content of the expected disturbances. The 


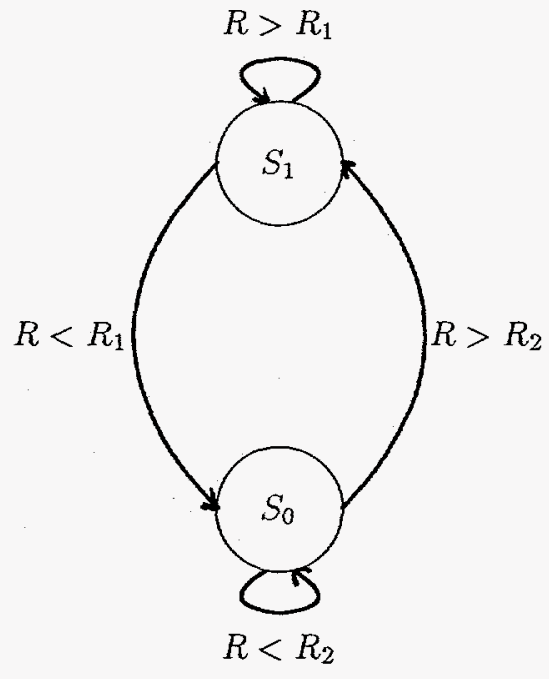

$$
\begin{gathered}
S_{0}=\text { pseudoinverse algorithm } \\
S_{1}=\text { heuristic algorithm }
\end{gathered}
$$

Figure 4: State Diagram of Algorithm Switching Hysteresis

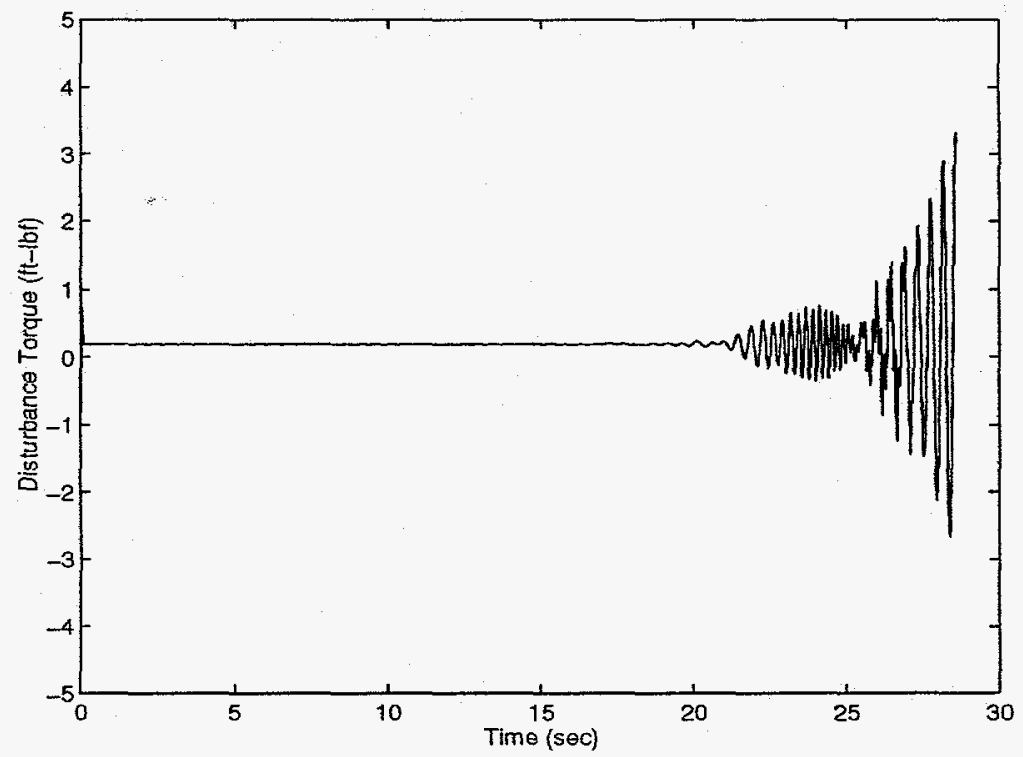

Figure 5: Disturbance Torque with All Masses in Same Location 


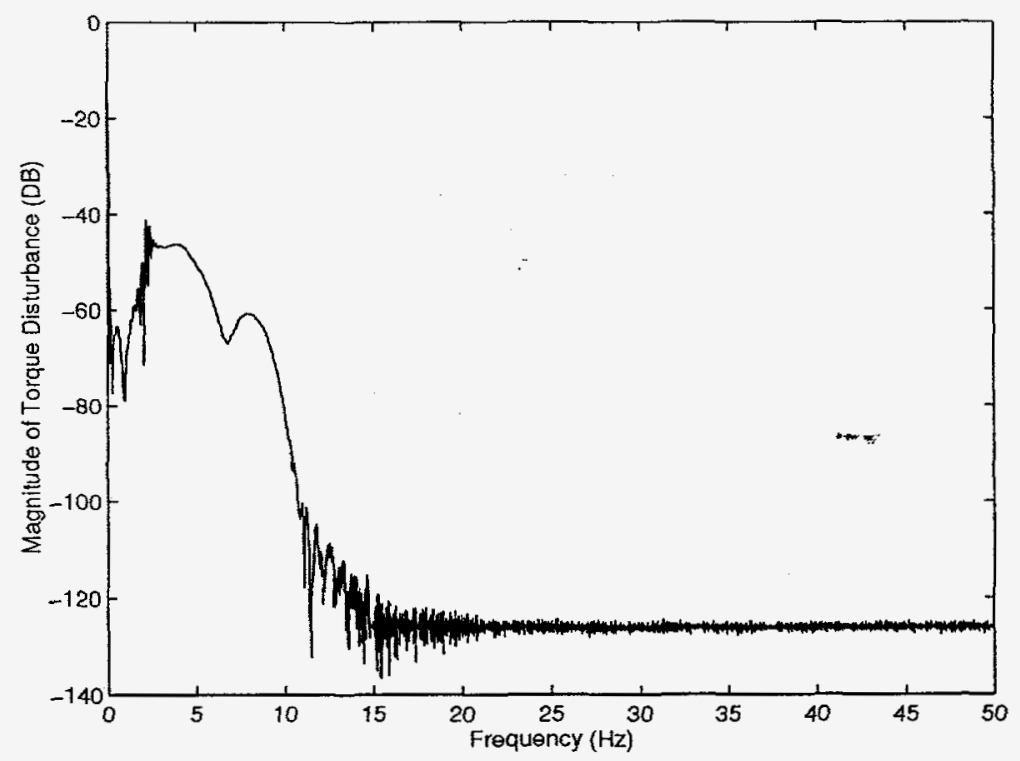

Figure 6: Frequency Content of Disturbance Torque

FFT of the disturbance torque is shown in Figure 6. The majority of the disturbance frequency content is below $5 \mathrm{~Hz}$, but a more conservative bound would be $10 \mathrm{~Hz}$. The next section presents a controller design using the disturbance characterization discussed in this section.

\section{Controller Design}

For the general closed-loop system shown in Figure 7, the transfer function from the disturbance $d(s)$ to the output $y(s)$ is given by

$$
\frac{y(s)}{d(s)}=\frac{p(s)}{1+p(s) c(s)}
$$

Equation (7) may be written as

$$
\frac{y(s)}{d(s)}=p(s) S(s)
$$

where the Sensitivity function $S(s)$ is defined as

$$
S(s)=\frac{1}{1+c(s) p(\varepsilon)}
$$




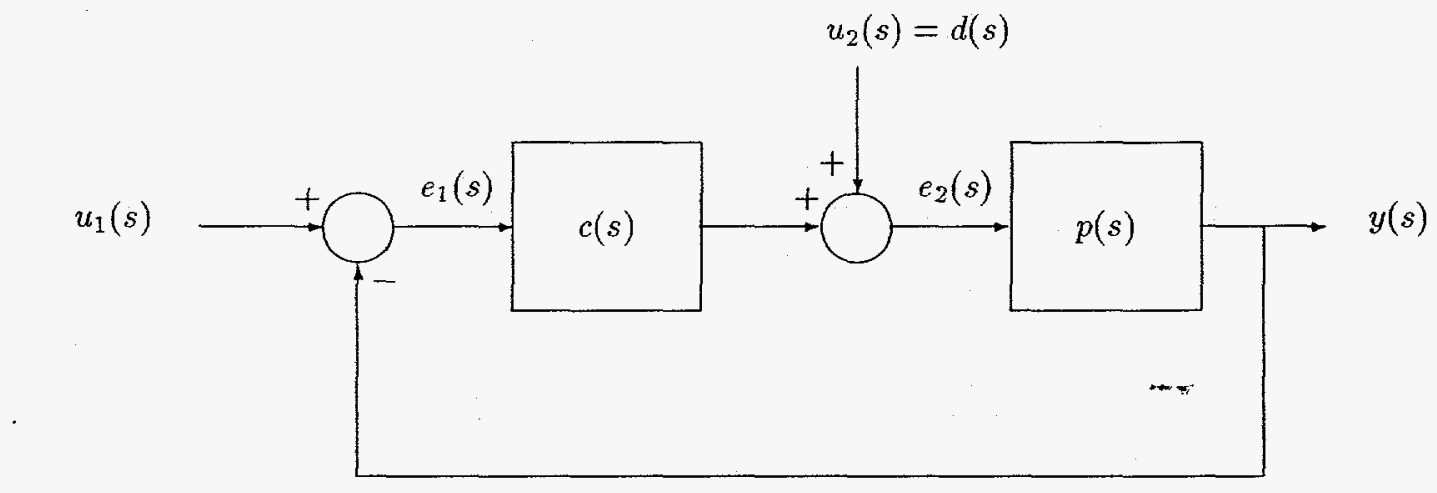

Figure 7: General closed-loop system

In order to minimize the effect of the disturbance on the output of the plant, it is desirable to minimize the Sensitivity function over the frequency range in which the disturbance is expected. One must also keep in mind that there is a trade off between the Sensitivity function $S(s)$ and the Complimentary Sensitivity function $T(s)$ shown below [6].

$$
S(s)+T(s)=1, \forall w, s=j w
$$

The controller structure shown in Figure 8 was chosen for the initial moving mass control system design. The controller in Figure 8 consists of an inner velocity loop and an outer position loop. It was assumed that position and velocity information would be available from sensors. Since all three masses will be identical, each mass will have an identical control system.

The goal of the moving mass control system is to maintain the desired mass positions in the non-rolling frame of the reentry vehicle. The angular velocity of the non-rolling frame, $w_{\text {rolling frame }}$, is an input to the velocity control loop. This is a feedforward term that helps the outer loop maintain the desired position in the non-rolling frame. The inner loop was designed to reject the expected torque disturbances, while the outer loop was designed to maintain the desired mass position with reasonable position errors. 


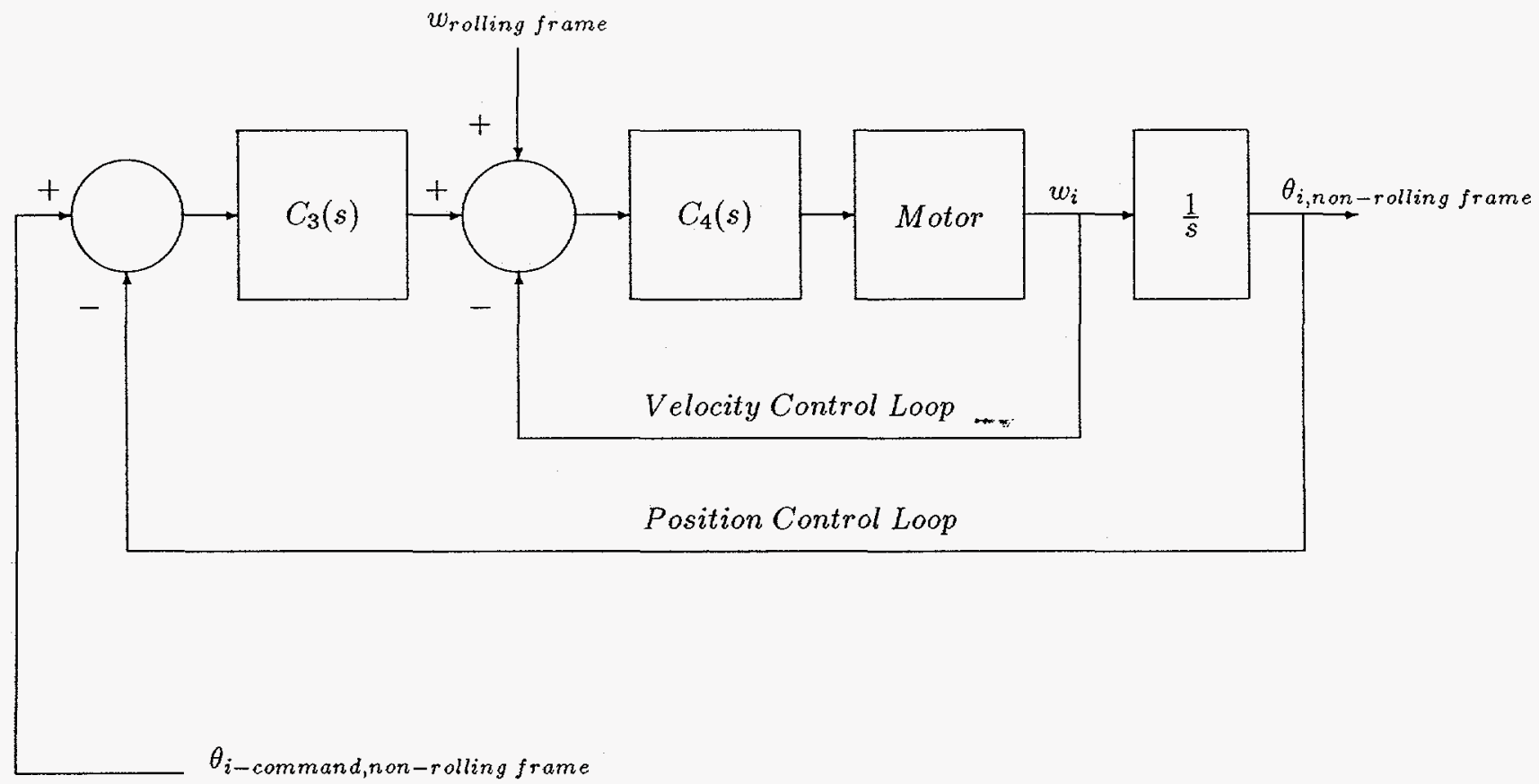

Figure 8: Moving Mass Control System Block Diagram

The disturbance rejection problem used in the design of the inner loop is shown in Figure 9. The input to the system is a commanded angular velocity $w_{\text {desired }}$. The controller $c(s)$ outputs a commanded current to the motor amplifier. It was assumed that a current amplifier with sufficient bandwidth would be used so that the amplifier dynamics would be negligible. The motor outputs a torque proportional to the motor torque constant $K_{T}$ and the gear ration $n$. The plant consists of the motor inertia and damping as well as the mass inertia and damping. For the preliminary design, the viscous damping $D$ will be assumed to be constant. Future efforts will focus on characterizing the damping as a function of axial acceleration. A brushless DC motor and gear reduction were chosen based on the expected torque requirements [7]. The transfer functions for the motor-mass system are shown in equation (11).

$$
\frac{1}{J s+D}=\frac{107.5}{s+1.398} \quad K_{T} n=1.432
$$

In order to ensure the desired disturbance rejection of the inner loop, one must first develop an estimate of the expected disturbances. From the previous section, a reasonable bound on the maximum expected torque 


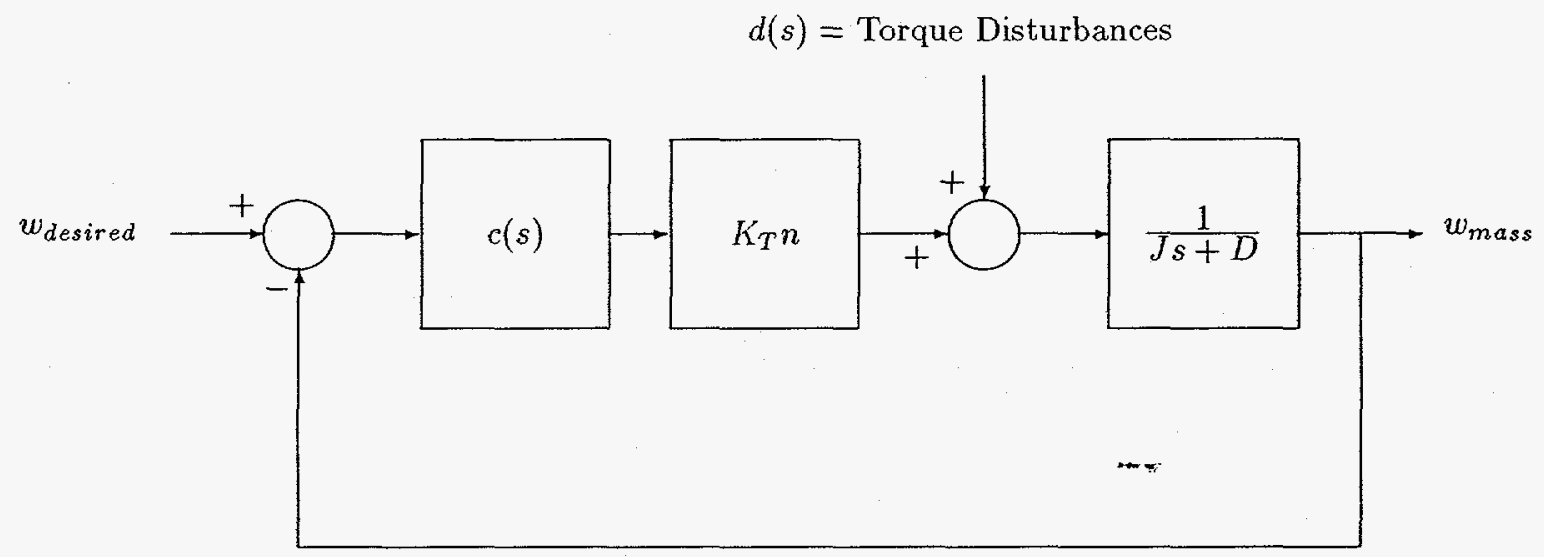

Figure 9: Moving Mass Control System, Velocity Inner Loop

disturbance is $3.5 \mathrm{ft}-l b f$. The bandwidth of this disturbance is assumed to be approximately $10 \mathrm{~Hz}$. It is desired to have the Sensitivity function attenuate the disturbance by 0.01 up to about $10 \mathrm{~Hz}$. A controller with a gain of 100 at low frequencies will meet this disturbance rejection requirement. In order to reduce the affect of higher frequency noise, the high frequency gain of the controller should be fairly low. A controller for the velocity loop, $C_{4}(s)$ in Figure 8 , which will meet the disturbance rejection requirement is shown in equation (12).

$$
C_{4}(s)=\frac{10 s^{2}+200 s+1000}{s^{2}+2 s+1}
$$

The velocity controller described by equation (12) yields an infinite gain margin with the simplified plant model used. However, if there is any additional phase lag in the system from unmodelled components, the controller could make the system marginally stable (a lower gain could cause the system to go unstable). Once an actual system is chosen, these issues can be addressed further.

A relatively simple PI (proportional - integral) position controller was implemented for $C_{3}(s)$ in Figure 8. The transfer function for $C_{3}(s)$ is shown in equation (13).

$$
C_{3}(s)=50 \frac{10 s+1}{s}
$$


Rate limiting was also added to $C_{3}(s)$ to limit the maximum commanded mass velocity to $+/-20 \mathrm{rad} / \mathrm{s}$. By limiting the maximum speed of the masses, disturbances to the RV's roll rate are minimized. This simplifies the task of holding the masses at the desired position in the non-rolling frame of the RV. Torque limiting was also included in the motor simulation to provide a more realistic result. Simulation results for the MMTC appear in the next section.

\section{Simulation Results}

This section presents simulation results for the MMTC. A nine degree-of-freedom simulation for a typical reentry vehicle was used to evaluate the performance of the MMTC. Six degrees-of-freedom describe the motion of the reentry vehicle. The rotation of each moving mass adds another degree-of-freedom. The simulation incorporates a rotating, ellipsoidal earth model and full aerodynamics including ablation effects.

For this simulation run, the commanded center of mass was $(-0.175,0)$ in the $(y, z)$ plane of the masses. The movement of the center of mass to the desired position is shown in Figures $10-12$.

The commanded mass positions for each of the three masses is shown in Figures 13-15. Because the commanded center of mass has a radius of less than $R_{1}$, these figures show the pseudoinverse algorithm.

The performance of the moving mass serva control system is shown in Figures 16-18. These figures show the error between the commanded and actual mass positions. The moving mass control system is very effective in maintaining the desired mass positions in the non-rolling frame of the RV, as shown by Figures 16-18. The mass position errors are fractions of a degree, even in the face of the large torque disturbances. 


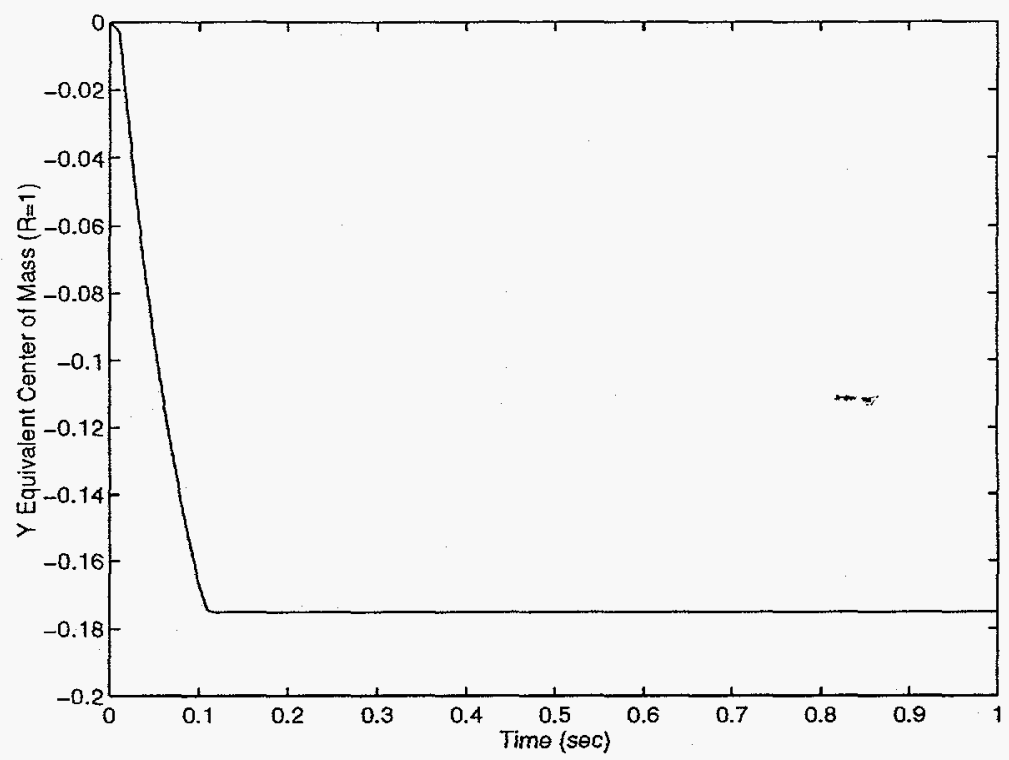

Figure 10: Equivalent Center of Mass - Y Axis, Expanded View

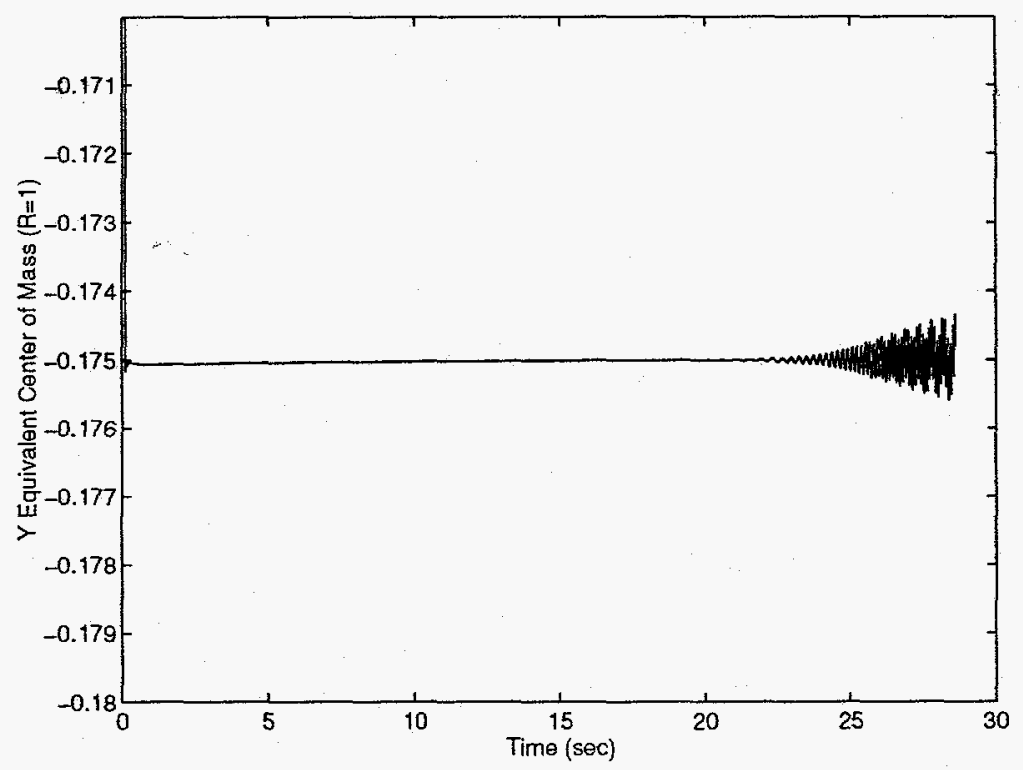

Figure 11: Equivalent Center of Mass - Y Axis 


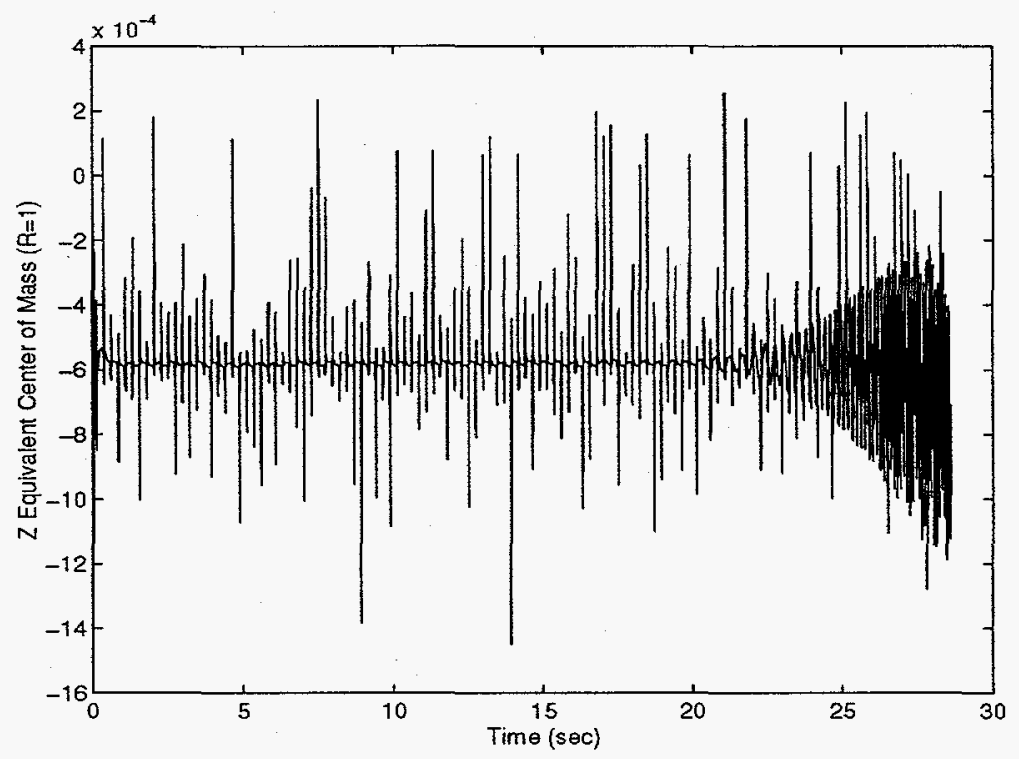

Figure 12: Equivalent Center of Mass - $\mathrm{Z}$ axis

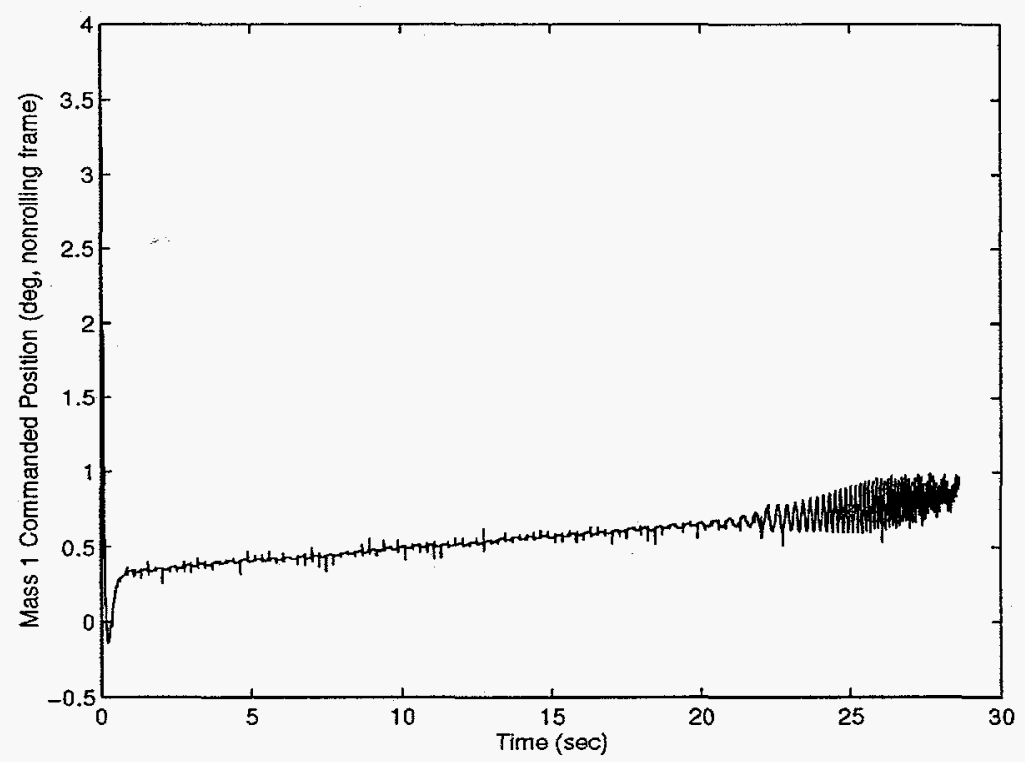

Figure 13: Mass 1 Commanded Position, Degrees in Non-Rolling Frame 


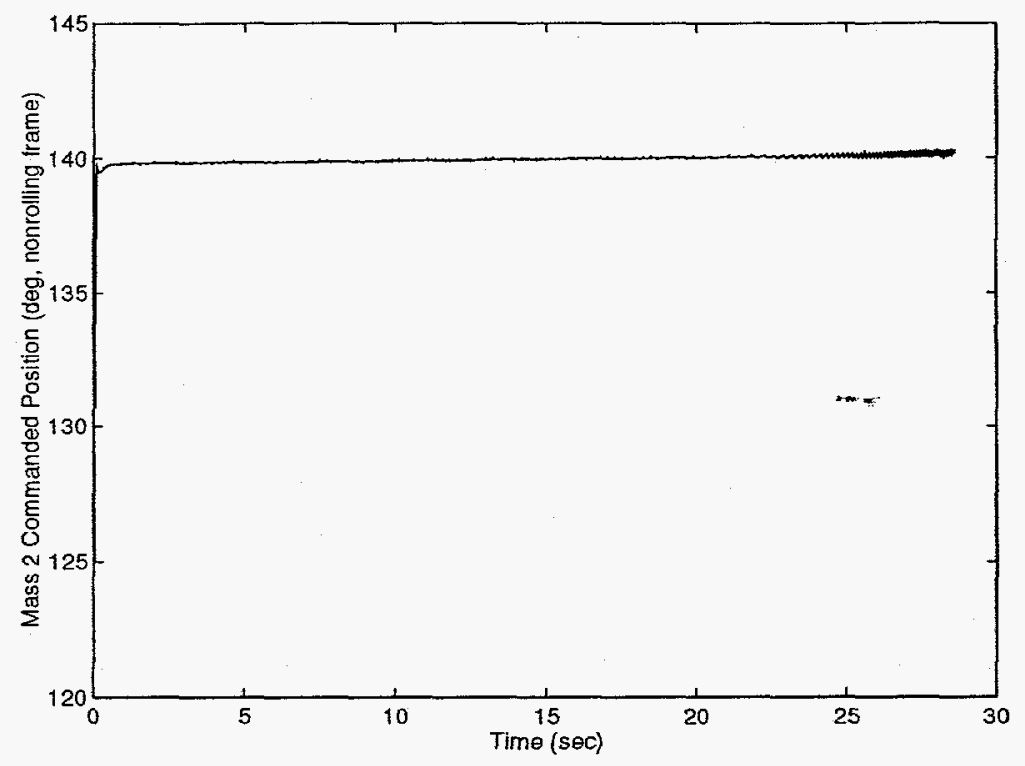

Figure 14: Mass 2 Commanded Position, Degrees in Non-Rolling Frame

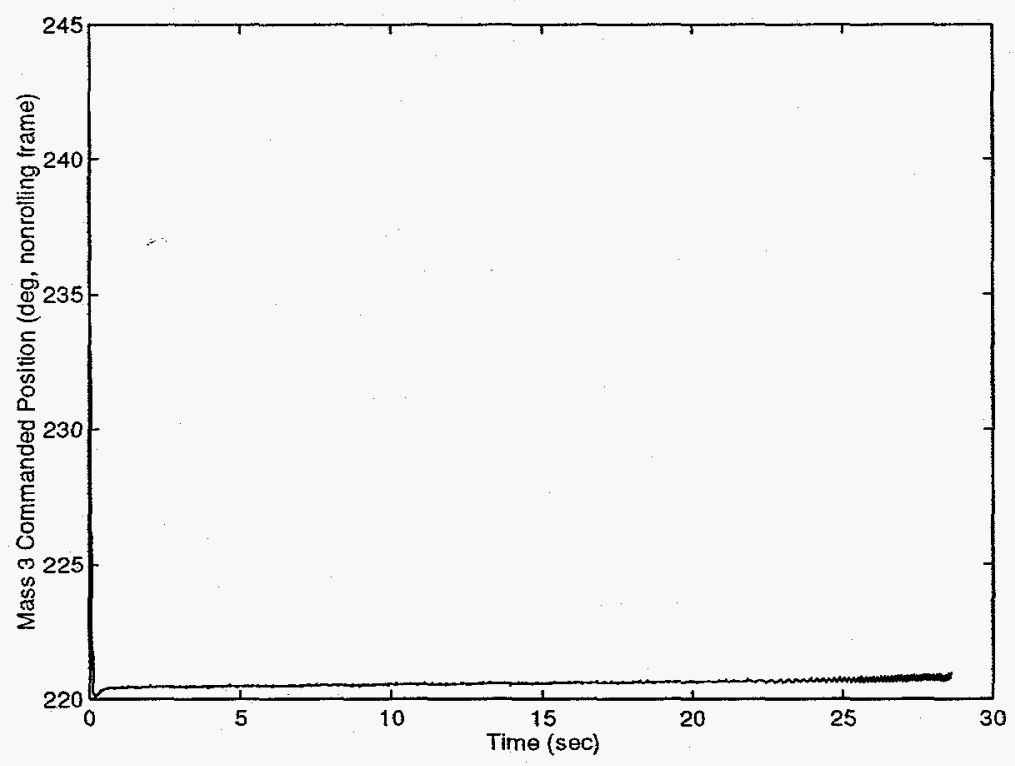

Figure 15: Mass 3 Commanded Position, Degrees in Non-Rolling Frame 


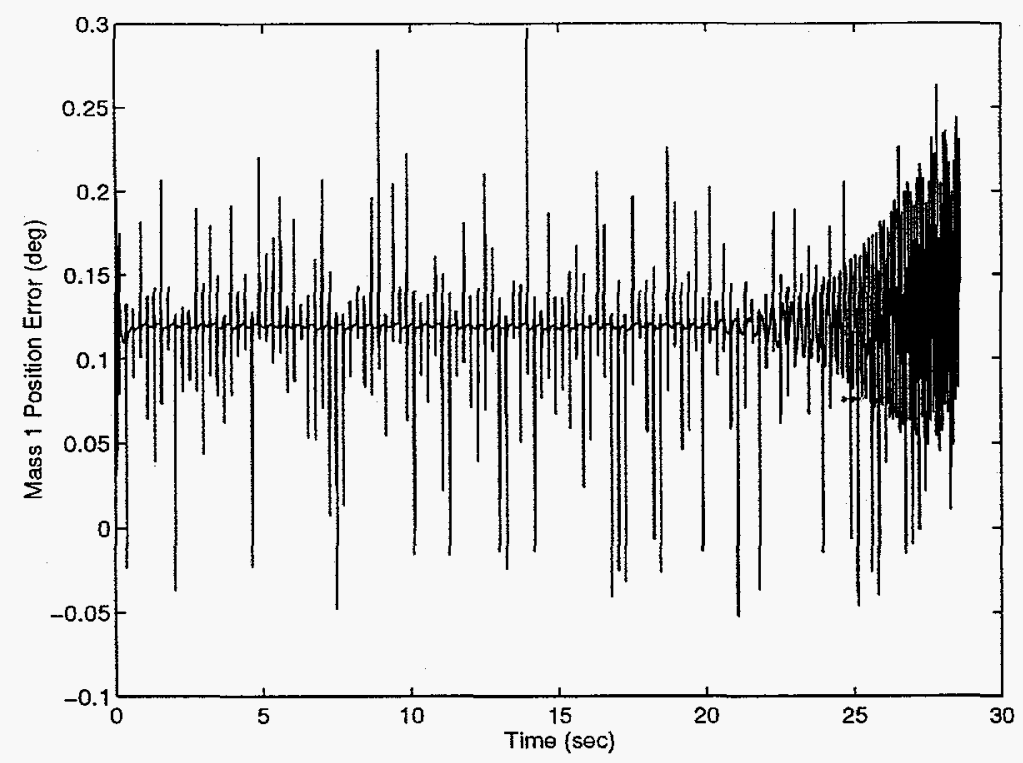

Figure 16: Mass 1 Position Error, Degrees

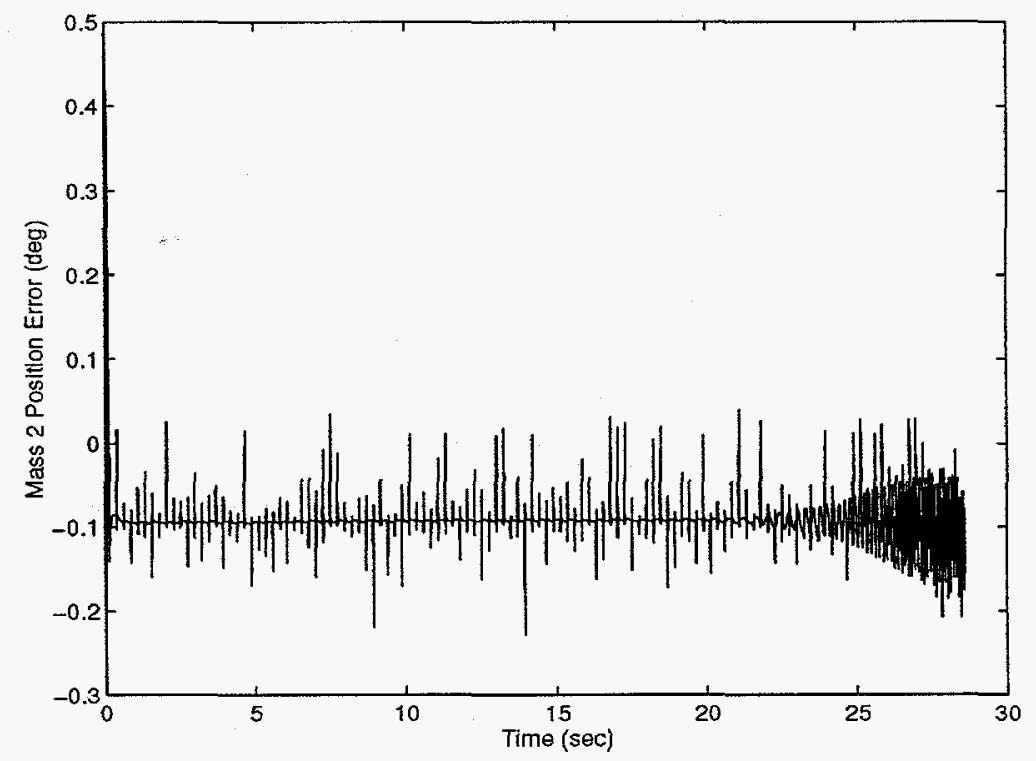

Figure 17: Mass 2 Position Error, Degrees 


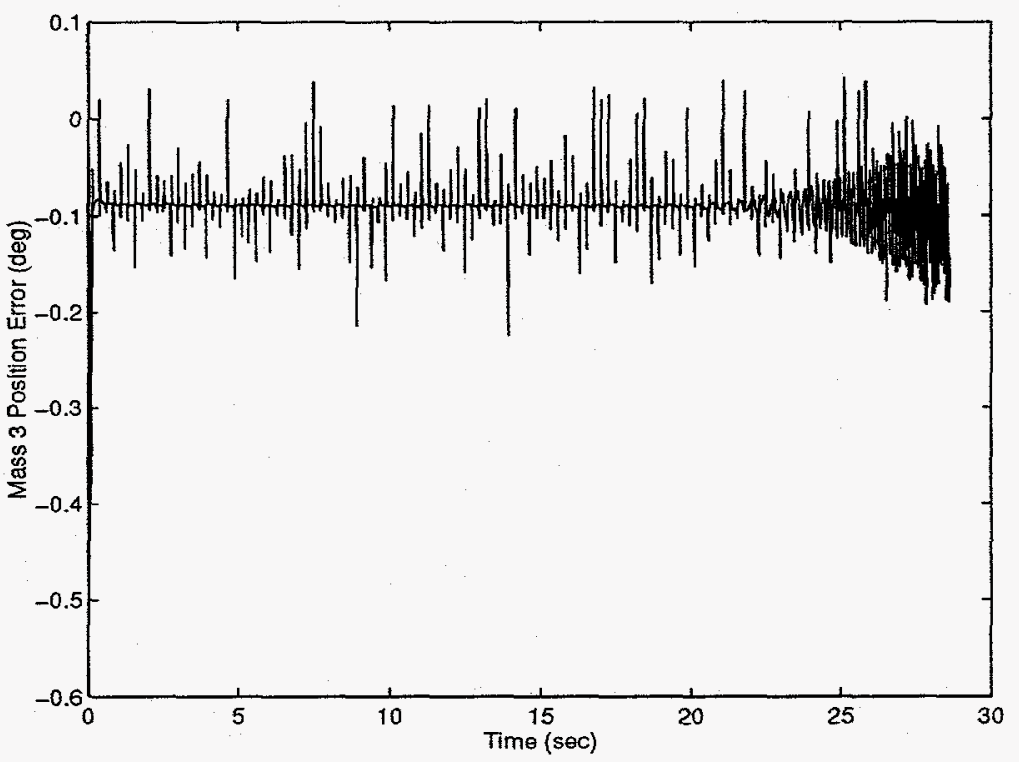

Figure 18: Mass 3 Position Error, Degrees 


\section{Summary and Conclusions}

This paper presented a preliminary design of a MMTC system for a maneuvering axisymmetric reentry vehicles. An algorithm was formulated for determining the positions of three equal masses that will generate a desired equivalent center of mass. Although a three mass system requires more hardware than a two mass system, a two mass system can require large mass movements to obtain a small change in the equivalent center of mass whereas a three mass system avoids these difficulties. The moving mass servo control system that must keep the masses in the desired positions can be subjected to large disturbance torques. These disturbance torques were characterized in both amplitude and frequency content. This information was then used to design a closed-loop mass position controller using classical frequency-domain techniques. Simulation results were then presented for the moving mass servo control system.

Future research will focus on alternate controller designs and the incorporation of the moving mass servo control system into a closed-loop navigation system. The design presented in this paper did not incorporate friction and sensor noise in the moving mass servo controller. Later, the motor and bearing friction will be modelled as a function of axial acceleration to verify that these types of disturbance torques do not have an

effect on the servo controller performance. Noise will also be added to the position and velocity sensors to determine their effect on the controller performance.

Acknowledgments: John Phelan provided invaluable assistance in formulating the mass position algorithm, and developed early versions of the final algorithm presented in this paper.

\section{References}

[1] F. J. Regan, Re-entry Vehicle Dynamics. AIAA Education Series, American Institute of Aeronautics and Astronautics, 1984.

[2] R. D. Tucker and A. F. Veneruso, "Analysis of a proposed movable mass reentry vehicle roll control 
system," Tech. Rep. SC-RR-710090, Sandia National Laboratories, Albuquerque, NM, March 1971.

[3] T. Petsopoulos and F. J. Regan, "A moving-mass roll control system for a fixed-trim re-entry vehicle," in Proceedings of the 32nd Aerospace Sciences Meeting and Exhibit, AlAA-94-0033, (Reno, NV), January 1994.

[4] R. D. Robinett, B. Rainwater, and S. A. Kerr, "Moving mass trim control for aerospace vehicles," in Proc. of the Annual AIAA Missile Sciences Conference, 1994.

[5] G. Strang, Linear Algebra and its Applications. Harcourt Brace Jovanovich: Addison-Wesley Publishing Company, 3 ed., 1988.

[6] J. M. Maciejowski, Multivariable Feedback Design. Wokingham, England: Addison-Wesley Publishing Company, 1989.

[7] "Brushless DC motors selection guide." Hathaway Motion Control Product Literature, 1994.

\section{DISCLAIMER}

This report was prepared as an account of work sponsored by an agency of the United States Government. Neither the United States Government nor any agency thereof, nor any of their employees, makes any warranty, express or implied, or assumes any legal liability or responsibility for the accuracy, completeness, or usefulness of any information, apparatus, product, or process disclosed, or represents that its use would not infringe privately owned rights. Reference herein to any specific commercial product, process, or service by trade name, trademark, manufacturer, or otherwise does not necessarily constitute or imply its endorsement, recommendation, or favoring by the United States Government or any agency thereof. The views and opinions of authors expressed herein do not necessarily state or reflect those of the United States Government or any agency thereof. 


\section{DISCLAMIER}

Portions of this document may be illegible in electronic image produets- Images are produced from the best available original docenment. 